\title{
Special issue on "Immunotherapy of cancer: future possible therapy for metastatic cancer"
}

\author{
Pravin D. Potdar \\ Faculty \& Professor of Stem Cell \& Genomic Research, Dr. A.P.J. Abdul Kalam Educational \& Research Centre, Prabhadevi, Mumbai \\ 400025, India.
}

Correspondence to: Prof. Pravin D. Potdar, Faculty \& Professor of Stem Cell \& Genomic Research, Dr. A.P.J. Abdul Kalam Educational \& Research Centre, Prabhadevi, Mumbai 400025, India. E-mail: ppravin012@gmail.com

How to cite this article: Potdar PD. Special issue on "Immunotherapy of cancer: future possible therapy for metastatic cancer". J Cancer Metastasis Treat 2020;6:38. http://dx.doi.org/10.20517/2394-4722.2020.75

Received: 22 Jul 2020 Accepted: 22 Jul 2020 Published: 27 Sep 2020

Academic Editor: JCMT Editorial Office Copy Editor: Cai-Hong Wang Production Editor: Jing Yu

The global burden of cancer incidence is estimated to be up to 18.1 million people with 9.6 million deaths in 2018. The major cause of death is due to the high toxicity of chemotherapeutic drugs and drug resistance developed by cancer cells. Thus, there is an unmet need for appropriate therapy which can kill cancer cell alone and reduce drug resistance. Immunotherapy is an alternative therapy for cancer which mainly boosts the immune response of these patients and kills cancer cells alone with fewer side effects. Since Professor James Allison from M.D. Anderson Cancer Centre, Texas, USA, received the Noble prize for his outstanding contribution to the field of immunotherapy, there has been great excitement in this field. Immunotherapy has become a powerful branch of cancer therapy for the treatment of various types of cancers due to milder side effects than the presently available chemotherapy or radiotherapy. It mainly works by strengthening the host immune responses against tumor cells by supplementation of modified immune system components. There are four different types of immunotherapies: monoclonal antibodies, cancer vaccines, immune checkpoint inhibitors, and Chimeric Antigen Receptor-T cell therapies. Immunotherapy can be given in combination with mild doses of chemotherapy or radiotherapy obtain effective and non-toxic treatment for most cancer patients.

With the development of several innovative technologies in the field of cancer medicine, molecular biology, stem cell biology, and bioinformatics, there has been a rapid increase in our understanding of the human immune system. Similarly, there is an extensive program planned to discover small molecules, peptides, recombinant antibodies, vaccines, and cellular therapeutics, which are being applied to manipulate the immune response of cancer patients to cure cancer. These immune therapies have provided significant

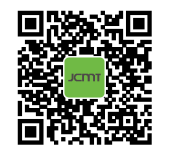


benefits against cancer, especially the application of immune checkpoint inhibitors, cell therapies, and vaccines. In this Special Issue on immunotherapy of cancer, we have invited pioneer scientists and clinicians involved in the latest work in this area to contribute their original manuscripts, case reports, clinical studies, and reviews that focus on immunotherapy for curing cancer.

There is an interesting paper written by Dr. Thibault Gauduchon from Lyon France, who explained that there is a great improvement in the cure of squamous cell carcinoma of head and neck cancer by changing the therapeutic management with immunotherapy using various checkpoint inhibitors or small molecules. They further highlighted that the checkpoint inhibitors are the first to be validated in secondline treatment with programmed death-1 and programmed death-ligand 1 (PD-L1) inhibitors to increase their effectiveness. The major goal of this paper by Dr. Gauduchon et al. is to provide an update on the possibilities of current immunotherapy in the management of Head and Neck Squamous Cell Cacinoma.

Snaar-Jagalska et al. from Leiden University, Netherlands, demonstrated the importance of inhibition of CXCR4 signaling in cancer metastasis by using the zebrafish xenograft model as a translational tool for anti-cancer discovery. This is a very important paper for scientists who are working on experimental cancer biology for drug discovery programs in cancer immunotherapy. Finally, they addressed the translational impact of targeting CXCR4 signaling in the tumor microenvironment for the treatment of metastatic cancer.

Matthew Spector et al. from the University of Michigan, Michigan, USA, reviewed novel immunotherapeutic approaches in head and neck cancer. They further stated that pembrolizumab is now approved as firstline immunotherapy for head and neck cancer. However, there are still low response rates in the cure of this cancer and therefore additional strategies need to be implemented. This review summarizes the most common immune disruptions, checkpoint inhibitors identified in head and neck cancer, and discusses ongoing approaches aimed at targeting the tumor immune microenvironment.

Professor Giovanni LO Re, a well-known oncologist from CRO Oncology Pordenone Aviano, Italy, documented his experience with an exceptional young inoperable patient for LA head metastatic pancreatic ductal adenocarcinoma (PDAC), treated with seven cycles of Nab-Paclitaxel-Gemcitabine (Nab-PCT-GEM) and RT 50 Gy/15 fractions combined to biweekly GEM and salvage FOLFIRINOX having five-year survival. He further stated that this is because this patient has travelled 15,000 km on foot, suggestive that his "walking therapy" may have helped this PDAC patient to survival more time. Dr. Giovanni well explained how a physical activity such as walking has helped in boosting the immunity of PDAC patients and increased survival rate. It is an interesting observation to note for many oncologists who are treating PDAC and other types of cancer: by implementing this simple strategy in their patients, they may have better survival.

In this Special Issue, we are lucky to have received a paper from Professor Porunelloor A. Mathew from UNT Health Centre, Texas, USA, who reviewed his experience working on Lectin-Like transcript 1 (LLT1) as an immunotherapeutic target for triple-negative breast and prostate cancer. Dr. Mathew suggested that blocking inhibitory signals to NK cells using monoclonal antibodies to LLT1 could enhance the lysis of prostate cancer and triple-negative breast cancer (TNBC) cells by NK cells. His lab further demonstrated that higher expression of LLT1 in TNBC and prostate cancer cells increased lysis of cancer cells after blocking LLT1 with monoclonal antibodies and may offer a potential target for breast and prostate cancer treatment. I hope this will be useful to many of our special issue readers when implementing this work in the cure of prostate and triple-negative breast cancers.

In this special issue, we included an article from Professor Kimberly Webster, a Medical Social Science expert from Northwestern University, Feinberg School of Medicine, USA who developed the Functional 
Assessment of Chronic Illness Therapy (FACIT) item library with patient-reported adverse events associated with immune checkpoint modulators (ICM). This FACIT item library will allow clinicians to monitor the value of ICM treatment for patients with cancer by looking at the clinically relevant measurement of symptoms. Thus, the use of item selection from FACIT item library will be encouraged in clinical research and clinical practice evaluations.

We are fortunate to obtain a very interesting review article on immune checkpoint inhibitor therapy in EBV-associated Gastric Cancer (EBVaGC) from Dr. Frank J. Slack from Beth Israel Deaconess Medical Centre, Harvard Medical School, Boston, USA. Several studies have shown that EBVaGC is commonly characterized by high lymphocytic infiltration in the tumor microenvironment, coupled with overexpression of immune checkpoint-related genes, including PD-L1. However, the regulation of PD-L1 expression in EBVaGC is poorly understood and further studies are needed to explain how EBV and host factors contribute to it. Dr. Slack highlighted that EBVaGC is a strong candidate for immune checkpoint blockade therapy after proper research in this area.

Thus, overall, we have to state here that this special issue on Immunotherapy of Cancer has included a number of original research articles, clinical studies, and systematic reviews of cancer immunotherapy from different angles contributed by various distinguished scientists and clinicians from prime institutes and medical centers all over the world. We hope that this special issue can provide valuable information to cancer researchers, clinicians, and academic professors for the enhancement of knowledge in the field of immunotherapy as well as better therapy of metastatic cancers.

\section{DECLARATIONS}

\section{Acknowledgments}

We would like to thank all the authors of this special issue for their excellent research stories and stimulating ideas. We would also like to express our great appreciation to all the special issue reviewers and editors, whose efforts substantially contributed to the improvement of the overall quality of this issue.

\section{Authors' contributions}

Wrote and reviewed the manuscript: Potdar PD

\section{Availability of data and materials}

Not applicable.

\section{Financial support and sponsorship}

None.

\section{Conflicts of interest}

The author declared that there are no conflicts of interest.

\section{Ethical approval and consent to participate}

Not applicable.

\section{Consent for publication}

Not applicable.

\section{Copyright}

(c) The Author(s) 2020. 